Published in final edited form as:

J Am Geriatr Soc. 2014 July ; 62(7): 1415. doi:10.1111/jgs.12875.

\title{
Response to Paul Regal
}

Eva M. Schmitt, PhD, Sharon K. Inouye, MD, MPH, and SAGES Study Group Hebrew SeniorLife - Aging Brain Center, Boston, Massachusetts

\section{To the Editor}

We appreciate this opportunity to clarify important aspects of our study (1) in response to the letter from Dr. Paul Regal. First, we would like to emphasize that severe hearing impairment was an exclusionary criteria for the SAGES study. Some study patients did use hearing aids, and were encouraged to use these during the interviews. In addition, the interviewers were carefully trained in communicating with older, hearing impaired patients and had portable amplifiers available for use as needed.

Since baseline dementia was an exclusionary criterion for this study, we followed a careful, multi-step process to rule out dementia, as follows: self or clinician report of a dementia diagnosis, medical record diagnosis of dementia, or baseline (preoperative) Modified MiniMental State (3MS) score $<69$ (or its education-adjusted equivalent) (2). Additional patients were excluded for possible dementia after neuropsychological testing and clinical adjudication of all study results by an expert panel. It is important to note that the Confusion Assessment Method (CAM) was only used for identification of delirium in the study, and was not utilized as part of the dementia diagnosis process nor for the course (improvement/ worsening) or trajectory of post-operative cognitive decline. Long-term cognitive decline in this study is measured with the complete neuropsychological test battery which is completed at $1,2,6,12$, and every 6 months thereafter. One of our primary outcome measures for cognitive functioning is our composite measure based on the neuropsychological test battery, which we refer to as our General Cognitive Performance score $(3,4)$. While no studies have yet been published with this database examining reversibility of delirium, the SAGES study does have a wide range of delirium instruments to use for this purpose, including cognitive screening tests, digit spans, CAM, CAM-S, Delirium Symptom Interview (DSI), and the Memorial Delirium Assessment Scale (MDAS)(2).

We believe this study provides a valuable resource for examination of risk factors for and outcomes of postoperative delirium in older persons.

Contact: Eva Schmitt, Hebrew SeniorLife - Aging Brain Center, 1200 Centre St 1200 Centre St., Boston Massachusetts 02131, T: 617-971-5390, F: 617-971-5309, EvaSchmitt@ hsl.harvard.edu.

Author Contributions: Both authors contributed to this paper.

Conflict of Interest: None of the authors report any conflicts of interest. All co-authors fully disclose they have no financial interests, activities, relationships and affiliations. The co-authors also declare they have no potential conflicts in the three years prior to submission of this manuscript.

Sponsor's Role: None. 


\section{ACKNOWLEDGMENTS}

Grant funding: Supported by Grants No. P01AG031720 (SKI) from the National Institute on Aging.

\section{REFERENCES}

1. Schmitt EM, Saczynski JS, Kosar CM, et al. The Successful Aging after Elective Surgery (SAGES) Study: Cohort Description and Data Quality Procedures. J Am Geriatr Soc. 2015; 63:2463-2471. [PubMed: 26662213]

2. Schmitt EM, Marcantonio ER, Alsop DC, et al. Novel risk markers and long-term outcomes of delirium: The successful aging after elective surgery (SAGES) study design and methods. J Am Med Dir Assoc. 2012; 13:818, e811-810. [PubMed: 22999782]

3. Gross AL, Jones RN, Fong TG, et al. Calibration and validation of an innovative approach for estimating general cognitive performance. Neuroepidemiology. 2014; 42:144-153. [PubMed: 24481241]

4. Jones RN, Rudolph JL, Inouye SK, et al. Development of a unidimensional composite measure of neuropsychological functioning in older cardiac surgery patients with good measurement precision. J Clin Exp Neuropsychol. 2010; 32:1041-1049. [PubMed: 20446144] 